\title{
Influence of Different Polymer Belts on Lipid Properties in Nanodiscs Characterized by CW EPR Spectroscopy
}

\section{Authors}

Matthias Hoffmann, ${ }^{\mathrm{a}, \mathrm{b},{ }^{*}}$ Jana Eisermann, ${ }^{\mathrm{a}, \mathrm{c},{ }^{*}}$ Florian Arndt Schöffmann, ${ }^{\mathrm{a}, \mathrm{b},{ }^{*}}$ Manabendra Das, ${ }^{\mathrm{d}}$ Carolyn Vargas, $^{\mathrm{d}-\mathrm{g}}$ Sandro Keller, ${ }^{\mathrm{d}-\mathrm{g}}$ Dariush Hinderberger ${ }^{\mathrm{a}, \mathrm{b}, 1}$

anstitute of Chemistry, Physical Chemistry - Complex Self-Organizing Systems, Martin Luther University (MLU) Halle-Wittenberg, Von-Danckelmann-Platz 4, 06120 Halle (Saale), Germany

bInterdisciplinary Research Center HALOmem, Charles Tanford Protein Center, MLU HalleWittenberg, Kurt-Mothes-Str. 3a, 06120 Halle (Saale), Germany

'Department of Chemistry - Molecular Sciences Research Hub, Imperial College London, 82 Wood Ln, W12 OBZ London, United Kingdom

${ }^{\mathrm{d} M o l e c u l a r ~ B i o p h y s i c s, ~ T e c h n i s c h e ~ U n i v e r s i t a ̈ t ~ K a i s e r s l a u t e r n ~(T U K), ~ E r w i n-S c h r o ̈ d i n g e r-S t r . ~ 13, ~} 67663$ Kaiserslautern, Germany

'Biophysics, Institute of Molecular Biosciences (IMB), NAWI Graz, University of Graz, Humboldtstr. 50/III, 8010 Graz, Austria

${ }^{f}$ Field of Excellence BioHealth, University of Graz, Graz, Austria

${ }^{g}$ BioTechMed-Graz, Graz, Austria

* these authors contributed equally

${ }^{1}$ Corresponding author

\begin{abstract}
With this study we aim at comparing the well-known lipid membrane model system of liposomes and polymer-encapsulated nanodiscs regarding their lipid properties. Using differential scanning calorimetry (DSC) and continuous wave electron paramagnetic resonance (CW EPR) spectroscopy, we characterize the temperature-dependent lipid behavior within 1,2-dimyristoyl-sn-glycero-3phosphocholine (DMPC) liposomes and nanodiscs made from such liposomes by application of various polymers based on styrene-co-maleic acid (SMA), diisobutylene-alt-maleic acid (DIBMA), and styreneco-maleic amide sulfobetaine (SMA-SB), a new SMA-derived copolymer containing sulfobetaine side chains. By incorporating a spin label doxyl moiety into the lipid bilayer in position 16 or 5 we were able to study the micropolarity as well as rotational restrictions onto the lipids in the apolar bilayer center and the chain region adjacent to the carbonyl groups, respectively. Our results suggest that all polymers broaden the main melting transition of DMPC, change the water accessibility within the lipid bilayer, and exhibit additional constraints onto the lipids. Independent of the used polymer, the rotational mobility of both spin-labeled lipids decreased with DIBMA exerting less restraints probably due to its aliphatic side chains. Our findings imply that the choice of the solubilizing polymer has to be considered an important step to form lipid nanodiscs which should be included into research of lipid membranes and membrane proteins in the future.
\end{abstract}

\section{Keywords}


nanodisc; CW EPR; SMA (styrene/maleic acid); DIBMA (diisobutylene/maleic acid); SMA-SB

(styrene/maleic sulfobetaine)

\section{Introduction}

Researchers have access to a variety of model membrane systems for lipid and protein studies [1-4]. One rather new model system consists of polymer-encapsulated nanodiscs, which are also called native nanodiscs or simply polymer/lipid particles. Some polymers such as poly-(styrene-co-maleic acid) $(\mathrm{SMA})[1,5]$ or poly-(diisobutylene-alt-maleic acid) (DIBMA)[6] are able to directly solubilize membrane lipids from liposomes or even cell lysates [1, 2, 4, 7]. However, research in this field is clearly application-driven, whereas fundamental differences between nanodiscs and liposomes or among nanodiscs made from different polymers are understood only in part. Most efforts aim towards improving the solubilizing properties of existing polymers [8-12], synthesizing new, more efficient or more gentle polymers [13-15], or facilitating the study of membrane proteins $[5,16]$. Initial fundamental research has been performed on the influence of the polymer on lipid properties, revealing e.g., a strong impact of SMA on the melting temperature of 1,2-dimyristoyl-sn-glycero-3phosphocholine (DMPC) $[6,17]$. Moreover, in a previous study, we could verify that the specific structure of the respective polymer affects its interaction pattern with small guest molecules (in the case at hand, nitroxide radicals), a finding which can be translated to lipid molecules [15]. This aspect highlights the question of how the polymer interacts with different parts of the lipids such as the headgroup, the interfacial carbonyl/glycerol region, and the apolar acyl chains, all of which is poorly understood up to now.

In this work, we employ two spin-labeled lipids (see Scheme 1) combined with continuous-wave electron paramagnetic resonance (CW EPR) spectroscopy to obtain insights into nanoscopic lipid properties in DMPC liposomes and polymer-encapsulated nanodiscs containing a lipid-bilayer core composed of DMPC that is surrounded by SMA, DIBMA, or poly-(styrene-co-maleic amide sulfobetaine) (SMA-SB). The application of CW EPR spectroscopy in lipid research is well established for liposomes and other membrane model systems $[18,19]$. Hence, here it will be used to obtain information on the polarity, rotational mobility, and lipid ordering in nanodiscs. The nanoscopic view offered by CW EPR is complemented by dynamic light scattering (DLS), transmission electron microscopy (TEM), and differential scanning calorimetry (DSC). Finally, combination of temperature-dependent CW EPR measurements with DSC provides a broad view on lipid properties and is a promising combination of methods to yield valuable information for future nanodisc research. 

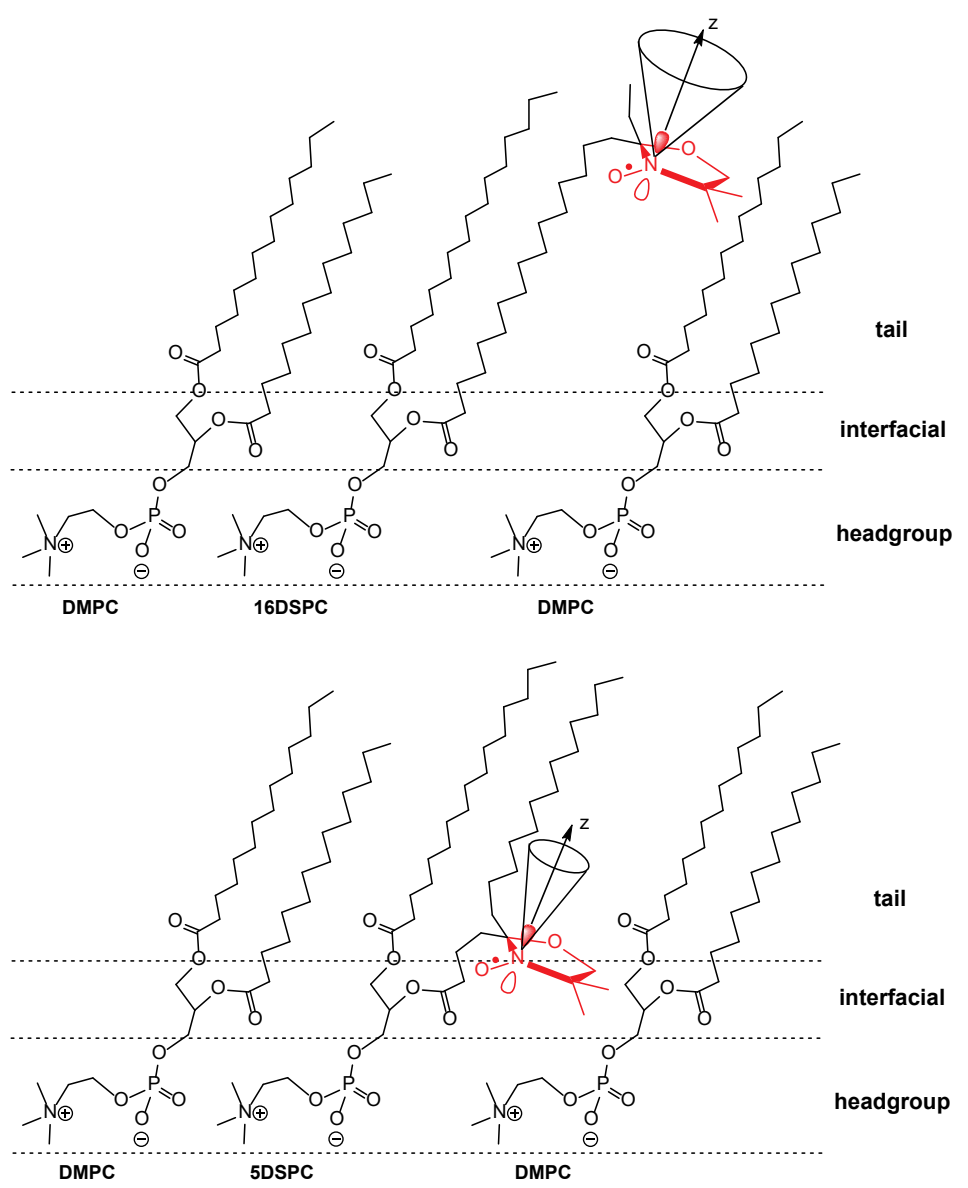

Scheme 1. Structures of 1-palmitoyl-2-stearoyl-(5-doxyl)-sn-glycero-3-phosphocholine (5DSPC) and 1palmitoyl-2-stearoyl-(16-doxyl)-sn-glycero-3-phosphocholine (16DSPC) incorporated into a DMPC lipid environment. The rotational mobility for the doxyl unit is represented for both 5DSPC and 16DSPC by a cone with a main axis that is parallel to the $z$-axis defined for the molecular frame of the doxyl's nitroxide group.

\section{Materials and Methods}

\section{Materials}

The three polymers poly-(styrene-co-maleic acid) (SMA 2.2:1 hydrolyzed from styrene/maleic anhydride (Xiran SZ30010)), poly-(diisobutylene-alt-maleic acid) (DIBMA) and poly-(styrene-co-maleic amide sulfobetaine) (SMA-SB) were synthesized as described elsewhere [15]. HPLC-grade chloroform as well as buffer salts were purchased from Carl Roth (Karlsruhe, Germany, Tris buffer salt $>99.3 \%$ and $\mathrm{NaCl}>99.7 \%$ ). 1,2-Dimyristoyl-sn-glycero-3-phosphocholine (DMPC) was obtained from Genzyme Pharmaceuticals (Cambridge, MA, USA). 1-palmitoyl-2-stearoyl-(5-doxyl)-sn-glycero-3-phosphocholine (5DSPC) and 1-palmitoyl-2-stearoyl-(16-doxyl)-sn-glycero-3-phosphocholine (16DSPC) were purchased from Avanti Polar Lipids (Alabaster, AL, USA).

\section{Preparation of polymer solutions and polymer/lipid samples}

Polymer solutions were prepared as described in [15]. An appropriate amount of each polymer was dissolved in saline Tris buffer ( $50 \mathrm{mM}$ Tris, $300 \mathrm{mM} \mathrm{NaCl}$, pH 7.4 in Millipore MilliQ water with a specific resistance of $\rho=18.2 \mathrm{M} \Omega \mathrm{cm}$ ) to yield mass concentrations of $25 \mathrm{mg} / \mathrm{mL}$ (SMA and DIBMA) or $15 \mathrm{mg} / \mathrm{mL}$ (SMA-SB) at room temperature followed by heating combined with ultrasonication for $30 \mathrm{~min}$ at $70^{\circ} \mathrm{C}$. After cooling down, all three polymer stock solutions were stable for at least two weeks if stored at room temperature. DMPC nanodiscs were prepared as follows: DMPC with added 
spin label lipid (5DSPC or 16DSPC, 2 mol\% of DMPC) was dissolved in chloroform. The solvent was evaporated in a gentle stream of nitrogen to yield a thin dry lipid layer. Tris buffer was added, and the concentration of DMPC was adjusted to somewhat above $1 \mathrm{mM}$. The lipids were allowed to suspend at $40{ }^{\circ} \mathrm{C}$ during ultrasonication of the sample for at least $10 \mathrm{~min}$. The resulting liposomes were extruded 35 times through a $100 \mathrm{~nm}$ polycarbonate membrane. Afterwards, polymer stock solutions were added to the thus prepared large unilamellar vesicles (LUVs) at a polymer/lipid ratio of 1:5 (mol:mol) and a lipid concentration of $1 \mathrm{mM}$. The molar mass of SMA-SB was estimated to be $4150 \mathrm{~g} \mathrm{~mol}^{-1}$ according to full conversion in its synthesis from SMA $\left(2700 \mathrm{~g} \mathrm{~mol}^{-1}\right)$. The mixtures of polymers and liposomes were then incubated at $30^{\circ} \mathrm{C}$ for $16 \mathrm{~h}$ while shaking.

\section{Dynamic light scattering (DLS)}

Dynamic light scattering (DLS) experiments were conducted on a Litesizer 500 (Anton Paar, Graz, Austria) with a $70 \mu \mathrm{L}$ Micro Quartz cuvette. The irradiation wavelength was $\lambda=658 \mathrm{~nm}$, while the detection angle was maintained at $90^{\circ}$ (side scattering) at constant temperature of $20^{\circ} \mathrm{C}$. Prior to each measurement, the sample was allowed to equilibrate for at least $1 \mathrm{~min}$. The data obtained from DLS measurements were evaluated in the Kalliope software (Anton Paar).

\section{Transmission electron microscopy (TEM)}

TEM samples were prepared by spreading $5 \mu \mathrm{L}$ of the (polymer-)lipid suspensions ( $20 \mu \mathrm{M}$ lipid) onto freshly glow discharged grids with carbon support film on copper (Quantifoil Micro Tools, Großlöbichau, Germany). After 30-45 s excess suspension was blotted up using filter paper. The grids were washed three times with MilliQ water. $5 \mu \mathrm{L}$ of $2 \%$ aqueous uranyl acetate solution was placed onto the grid and also blotted up after $1 \mathrm{~min}$. After preparation the samples were dried for at least $24 \mathrm{~h}$. All TEM samples were examined with a Zeiss EM 900 transmission electron microscope (Carl Zeiss Microscopy, Jena, Germany).

\section{Differential scanning calorimetry (DSC)}

For DSC, sample preparation was performed as described above using a higher amount of both lipid and polymer ( $5 \mathrm{mM}$ lipid target concentration in the samples). Prior to all measurements, the samples as well as the reference buffer solution was degassed under vacuum while stirring. DSC measurements were performed using a MicroCal VP-DSC differential scanning calorimeter (MicroCal, Northampton, MA, USA). In all experiments, 5 heating/cooling cycles were measured to assure sample stability and reproducibility from which one representative heating curve (heating rate $60 \mathrm{~K} \mathrm{~h}^{-1}$ between 2 and $70^{\circ} \mathrm{C}$ ) was chosen. Further evaluation of the DSC results involved subtraction of regularly collected buffer/buffer baselines as well as subtraction of offset values, if necessary.

\section{CW EPR spectroscopy}

X-band continuous-wave electron paramagnetic resonance (CW EPR) spectroscopic measurements were performed with the Miniscope MS5000 (magnettech, Freiberg Instruments, Freiberg, Germany) benchtop spectrometer. The connection to the $\mathrm{HO}$ temperature control unit (magnettech) allowed the recording of temperature series. All spectra were measured with the following settings: center field position of $337.5 \mathrm{mT}$, field sweep width of $10 \mathrm{mT}$, modulation amplitude of $0.1 \mathrm{mT}$ and a microwave power of $3.63 \mathrm{~mW}$. For sample preparation, 10-15 $\mu \mathrm{L}$ of the sample solution were filled into micropipettes (BLAUBRAND intraMark, Wertheim, Germany) and capped with capillary tube sealant (CRITOSEAL Leica, Wetzlar, Germany). Analysis of the recorded CW EPR spectra is based on simulations using MatLab (R2020b, v. 9.9) in combination with the EasySpin toolbox (v. 6.0.0 dev.26) for EPR spectroscopy [20]. Note that all spectra were simulated with a single component using the 
model of anisotropic Brownian motion with an axial symmetry and orienting potential (see SI for further information) [21, 22].

\section{Results}

\section{Preparation of nanodiscs}

The general formation of nanodiscs was confirmed with the aid of DLS and TEM, as exemplarily shown in Figure S1 and Figure S2. From DLS, it is evident that the liposomes were solubilized almost completely. After addition of polymer to the liposomes and incubation, all suspensions appeared clear. SMA and SMA-SB were more efficient in solubilizing DMPC than DIBMA. In samples containing DIBMAbased lipid particles (DIBMALPs), a small fraction of nonsolubilized liposomes remained. Interestingly, if applying the same polymer/lipid ratio for all three polymers, DIBMALPs clearly exhibit larger hydrodynamic diameters compared with SMALPs and SMA-SBLPS, which is in accordance with literature reports [6]. TEM observations support the DLS-derived findings. The presence of nanodiscs in each measured sample was confirmed and, in addition, the diameters of the detected nanodiscs correspond in their magnitude to the hydrodynamic diameters determined by DLS (see Figure S1).

\section{Temperature-dependent measurements of lipid properties in nanodiscs}

Temperature-dependent CW EPR spectroscopic (Figure 1) and differential scanning calorimetric measurements (DSC, Figure 2, top) of all lipid samples were performed as described above, and we first discuss the DSC data. The DSC trace of the DMPC liposomes was similar to that reported in the literature [23]. While all three polymers had a strong effect on the DSC baseline, no thermotropic transitions as reflected in DSC peaks were observed for polymer-only samples, that is, in the absence of lipid (see Figure S3). From the results shown in Figure $\mathbf{2 A}$ and $\mathbf{B}$ (top), it can be concluded that the lipid main transition broadens when polymer is added, as expected from the resulting decrease in the size of the cooperative unit [6]. In addition, it is evident that all three polymers had an effect on the main transition temperature $T_{\mathrm{m}}$ of DMPC: $T_{\mathrm{m}}$ was decreased in SMALPs and DIBMALPs, whereas SMASB led to a slight increase in $T_{\mathrm{m}}$.

\section{EPR-spectroscopic results}

The thermodynamic observations on the phase behavior of DMPC-based model membrane systems was complemented by a nanoscopic view gathered through CW EPR spectroscopy. To this end, we focused on the two spin-labeled lipids 16DSPC and 5DSPC, which allow characterizing the membrane center in the acyl chain region and the region close to the polar headgroup, respectively [18, 24-26]. Based on the position of the doxyl-unit along the fatty acid chain, temperature-dependent changes in the LUVs as well as nanodiscs were found through different simulation parameters (see Figure 1). 

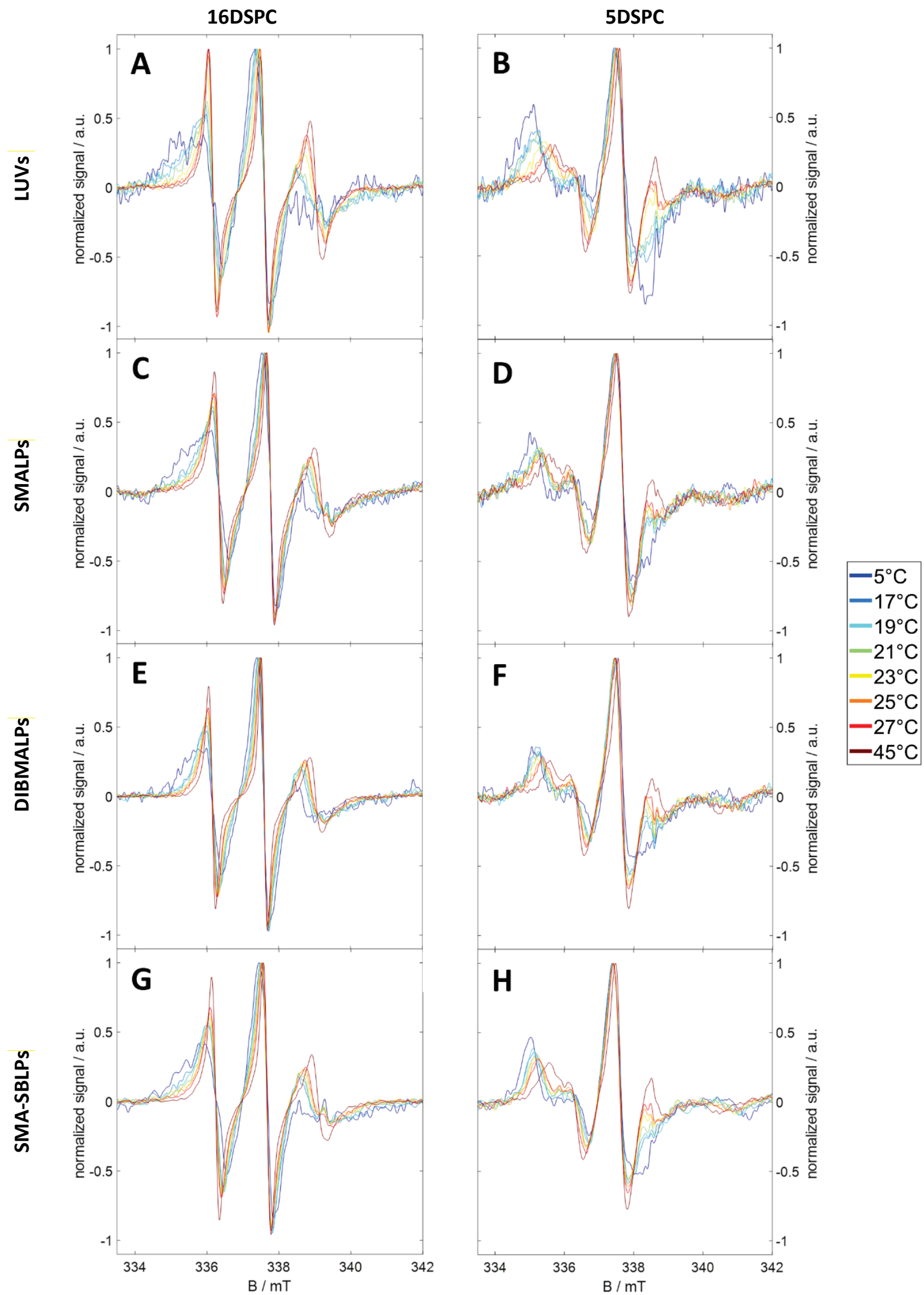

Figure 1. Temperature-dependent CW EPR spectra of 16DSPC (left column) and 5DSPC (right column) incorporated in DMPC LUVs or several polymer-encapsulated nanodiscs; (A/B) LUVs; (C/D) SMALPs; (E/F) DIBMALPs; (G/H) SMA-SBLPs. 
The line shape of the respective CW EPR spectra reflects the dynamics of the spin label. Three equally spaced sharp lines of similar intensity are associated with an unrestricted reorientational motion of the nitroxide. Upon increasing the spatial restriction on the mobility of the doxyl unit, the similarity of these three lines vanishes, while the apparent hyperfine splitting $a_{\text {iso,app }}$ increases (further described in the Discussion section and $\mathbf{S I}$ ), and the line width of the recorded spectral features rises. The CW EPR spectra become more anisotropic with (i) decreasing temperature for LUVs as well as nanodiscs and (ii) from the position of the doxyl label along the acyl chain: 5DSPC spectra re more anisotropic than 16DSPC spectra. The doxyl unit of 16DSPC is positioned at the end of the acyl chains as compared with 5DSPC that is close to the carbonyl region in the bilayer. Hence, 16DSPC shows higher reorientational mobility and, therefore, more isotropic spectra in all tested DMPC model membrane systems. Note that the CW EPR spectra of LUVs feature more isotropic peaks than those of the three nanodisc systems. A superficial, visual comparison of SMALPs, DIBMALPs, and SMA-SBLPs seems to indicate that the differences in the line shapes of the spin labels are insignificant.

Therefore, we performed spectral simulations using the model of anisotropic Brownian motion with axial symmetry (see $\mathbf{S I}$ for further information). Two important parameters that derive from this approach are (i) the rotational correlation time $\tau_{\mathrm{c}}$ and (ii) the segmental order parameter $S_{z z}$ for the doxyl unit. The respective values for both parameters at temperatures below $\left(15^{\circ} \mathrm{C}\right)$, around $\left(23^{\circ} \mathrm{C}\right)$, and above $\left(35^{\circ} \mathrm{C}\right)$ the main phase transition temperature $T_{\mathrm{m}}$ of DMPC are summarized in Table 1 and Table 2.

Table 1. CW EPR simulation results of 5DSPC spectra in DMPC LUVs and nanodiscs.

\begin{tabular}{|c|c|c|c|c|c|c|c|c|}
\hline \multicolumn{9}{|c|}{ 5DSPC } \\
\hline \multirow{2}{*}{$T /{ }^{\circ} \mathrm{C}$} & \multicolumn{2}{|c|}{ LUVs } & \multicolumn{2}{|c|}{ SMALPS } & \multicolumn{2}{|c|}{ DIBMALPS } & \multicolumn{2}{|c|}{ SMA-SBLPS } \\
\hline & $S_{z z}$ & $\tau_{\mathrm{c}} / \mathrm{ns}$ & $S_{z z}$ & $\tau_{\mathrm{c}} / \mathrm{ns}$ & $S_{z z}$ & $\tau_{\mathrm{c}} / \mathrm{ns}$ & $S_{z z}$ & $\tau_{\mathrm{c}} / \mathrm{ns}$ \\
\hline 15 & 0.66 & 27.7 & 0.70 & 21.3 & 0.70 & 27.4 & 0.70 & 26.6 \\
\hline 23 & 0.61 & 9.7 & 0.67 & 15.1 & 0.67 & 16.2 & 0.66 & 16.2 \\
\hline 35 & 0.57 & 5.9 & 0.63 & 8.6 & 0.61 & 8.3 & 0.62 & 7.9 \\
\hline
\end{tabular}

Table 2. CW EPR simulation results of 16DSPC spectra in DMPC LUVs and nanodiscs.

\begin{tabular}{c|cc|cr|rr|rr}
\hline \multicolumn{10}{c|}{ 16DSPC } \\
\hline \multirow{2}{*}{$T /{ }^{\circ} \mathrm{C}$} & \multicolumn{2}{|c|}{ LUVs } & \multicolumn{2}{c|}{ SMALPs } & \multicolumn{2}{c|}{ DIBMALPs } & \multicolumn{2}{c}{$S M A-S B L P S$} \\
& $S_{\mathrm{zz}}$ & $\tau_{\mathrm{c}} / \mathrm{ns}$ & $S_{\mathrm{zz}}$ & $\tau_{\mathrm{c}} / \mathrm{ns}$ & $S_{\mathrm{zz}}$ & $\tau_{\mathrm{c}} / \mathrm{ns}$ & $S_{\mathrm{zz}}$ & $\tau_{\mathrm{c}} / \mathrm{ns}$ \\
\hline $\mathbf{1 5}$ & 0.25 & 6.5 & 0.36 & 3.3 & 0.34 & 5.1 & 0.35 & 4.5 \\
$\mathbf{2 3}$ & 0.18 & 3.4 & 0.32 & 2.6 & 0.29 & 3.3 & 0.32 & 3.1 \\
$\mathbf{3 5}$ & 0.07 & 1.6 & 0.27 & 1.9 & 0.26 & 2.2 & 0.28 & 1.8 \\
\hline
\end{tabular}

The rotational correlation time $\tau_{\mathrm{c}}$ is derived from the diffusion tensor elements $D_{\|}$(motion around the unique axis of the NO-group) and $D_{\perp}$ (perpendicular to unique axis) as $\tau_{\mathrm{c}}=\frac{1}{6 \sqrt[3]{D_{\perp}^{2} D_{\|}}}$and is a simplified measure of the rotational mobility of the doxyl unit. As described above, changes in the spectral line shape reveal fastest motion for 16DSPC inside LUVs. The $\tau_{\mathrm{c}}$-values for the three nanodisc system are similar to but slightly higher than for LUVs above $T_{m}$, indicating a more restricted mobility of the nitroxide. The segmental order parameter $S_{z z}$ correlates with the semi-cone angle $\beta$ via $S_{\mathrm{zz}}=$ $\frac{1}{2}\left(3 \cos ^{2} \beta-1\right)[18]$. The semi-cone angle confines the wobbling motion of the respective observable segment based on the position of the doxyl-unit along the acyl chain of the lipid. A rigid crystal structure of the membrane would be described by the maximum value of $S_{\mathrm{zz}}=1$, whereas a state of maximum disorder would be characterized by its lowest value of $S_{\mathrm{zz}}=0$ [21]. The closer the doxyl unit 
is situated towards the headgroup region of the lipid bilayer, the larger the order parameter. When comparing LUVs and nanodiscs, SMALPs, DIBMALPs, and SMA-SBLPs yielded a higher order parameter than polymer-free liposomes. This effect was more pronounced for the 16DSPC spin label probing the center of the lipid bilayer than for the 5DSPC spin label near the carbonyl region.

\section{Discussion}

As shown in the results, we are able to correlate the temperature-dependent lipid characteristics detected by CW EPR with thermodynamic information obtained by DSC measurements. This enables us to get insight into the fundamentals of polymer/lipid interactions in nanodiscs which is much needed in this field of research. The successful preparation of DMPC nanodiscs with all three polymers is verified by DLS and TEM. In addition, DSC reveals that in all samples the lipid properties change as expected: (i) the main transition of DMPC broadens because of a decrease in the size of the cooperative unit reflecting a smaller particle size, and (ii) a small effect on $T_{m}$ of DMPC is observed for all polymers. At the polymer/lipid ratio studied here, no drastic decrease in $T_{\mathrm{m}}$ was observed, as is the case for SMALPs made from the more hydrophobic copolymer SMA(3:1) [6]. In case of SMA-SB, a very small fraction of remaining liposomes was detected in the DSC traces as a sharp peak on top of the broad main transition, which, however, was not observed in DLS measurements (see Figure 2A and B, top).

16DSPC

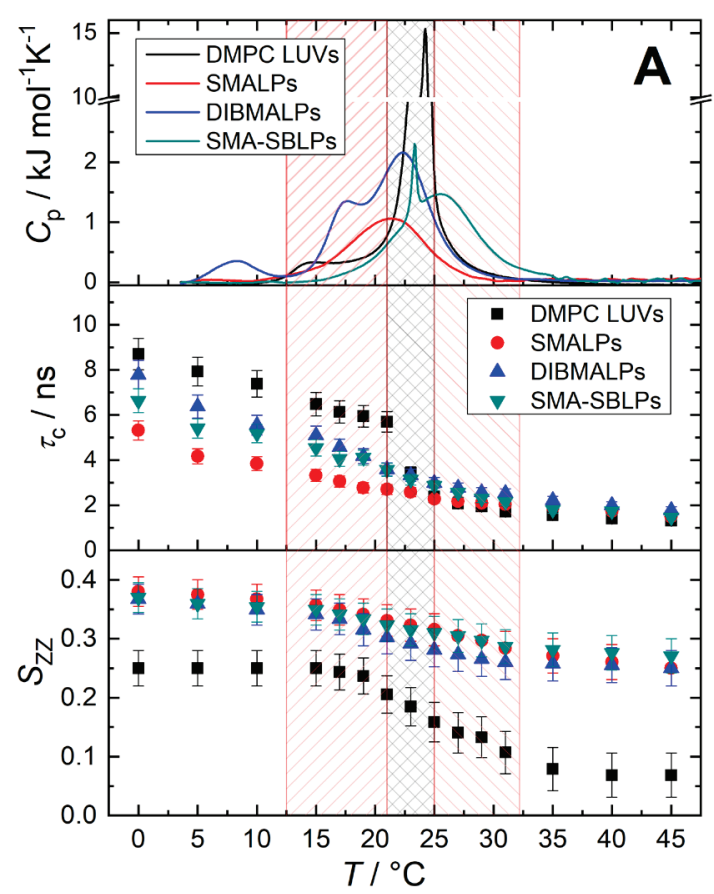

5DSPC

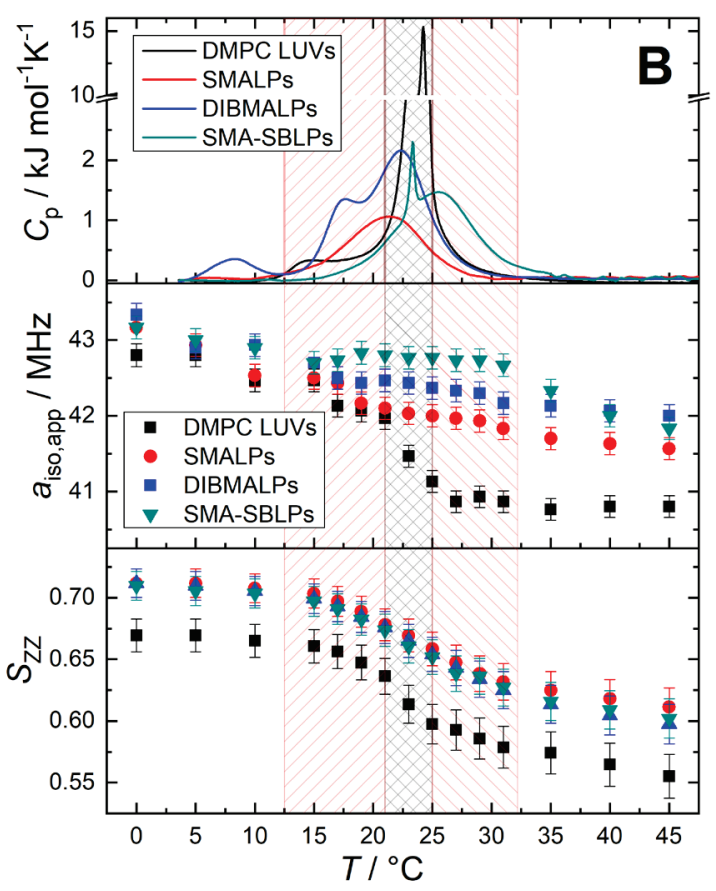

Figure 2. Comparison of the DSC thermograms of DMPC LUVs, SMALPs, DIBMALPs, and SMA-SBLPs with best-fit simulation parameters extracted from CW EPR measurements using (A) 16DSPC and (B) 5DSPC. Shown are the temperature dependencies of the rotational correlation time $\tau_{c}$, the mean apparent isotropic hyperfine coupling value $a_{\text {iso,app, }}$ and the nitroxide order parameter $S_{z z}$.

On the basis of the CW EPR results, a direct comparison of 16DSPC and 5DSPC for the same model membrane system yields a higher reorientational mobility for the doxyl unit placed at the end of the acyl chain (16DSPC), as highlighted in Scheme 1, leading to more isotropic spectra corresponding to decreased rotational correlation times $\tau_{c}$ (compare Figure 2A and Figure S3B). Note that the temperature range across which $\tau_{c}$ changes correlates with the broadness of the respective main transition peak determined by DSC. All three nanodisc systems exhibit a decreasing rotational mobility of 16DSPC above $T_{\mathrm{m}}$. Moreover, in a previous study of Stepien et al. focusing on nanodiscs encircled 
by membrane scaffold protein (MSP)[18], a more anisotropic motion of the doxyl unit was found through the diffusion tilt angle $\beta \mathrm{n} S_{z z}$ (discussed below). This trend was rationalized by the increased pressure exerted by the protein belt on the encircled lipid-bilayer patch. A similar effect found for the polymers studied here is depicted in Figure 3. For spin-labeled lipids, $\beta$ plays a crucial role as it significantly influences the shape of the CW EPR spectra [22] as well as the proportions for the diffusion tensor elements $D_{\|}$and $D_{\perp}$. 16DSPC possesses a smaller diffusion tilt angle in all three nanodiscs, which correlates with a more anisotropic motion of the respective doxyl unit.

Another feature already observed with MSP nanodiscs [18] is the change in the polarity profile, which can be characterized with the hyperfine coupling tensor $\mathbf{A}$ [27]. Its diagonal values are further used to calculate the apparent isotropic hyperfine coupling value $a_{\text {iso,app }}$ (as described in the SI) to analyze the hydrophobicity in the local environment of the doxyl unit throughout the complete temperature series. Note that especially the measurements below $T_{\mathrm{m}}$ do not represent isotropic CW EPR spectra, emphasizing the "apparent" character of the $a_{\text {iso }}$ values. In all DMPC nanodiscs, compared with LUVs, the water accessibility to the polar headgroup is significantly increased (schematically shown in Figure 3), reflected in a higher hyperfine coupling value in the liquid-crystalline phase (Figure 2B), whereas a hydrophobic barrier is formed in the center of the bilayer (see Figure S4A) below $T_{\mathrm{m}}$. Moreover, this water accessibility varies between the three different polymers SMA, DIBMA, and SMA-SB, as reflected in different interaction patterns between them and DMPC. This is particularly obvious in the case of SMA, which, as already shown in our previous study with small nitroxide radicals as guest molecules [15], induces a significant drop in the isotropic $g$-value for 16DSPC (see Fehler! Verweisquelle konnte nicht gefunden werden.A). Note that the observation of a hydrophobic barrier in the center of MSPbased nanodiscs cannot be applied in general to all polymer-encapsulated systems as shown by the behavior of $a_{\text {iso,app }}$ for 16DSPC (see Figure S4A), again indicating that the type of polymer affects the polarity profile of the membrane itself.

For $n$ DSPC spin-labels (with $n=5,16$ in our study), $S_{z z}$ reports only on the respective segment of the hydrocarbon chain to which the nitroxide fragment is attached. Thus, it is a segmental order parameter and does not reflect the order of the whole chain. The closer the doxyl unit is located to the polar headgroup of the lipid, the more tightly packed are their respective segments in the fatty acid chains (see Scheme 1). The decrease of $S_{z z}$ with increasing temperature correlates with the phase behavior determined via DSC independent from the respective model membrane system. Note that all tested nanodisc systems lead to an increase in the order parameter compared with DMPC liposomes. This effect is least pronounced for DIBMALPs (but still of the same magnitude as both SMALPs and SMASBLPs), which form larger nanodiscs and, consequently, contain a lower percentage of lipid molecules that are in vicinity to the polymer rim. Moreover, DIBMA displays weaker interactions with the attached spin probe, thus leading to a reduced change in $S_{z z}$ [21].

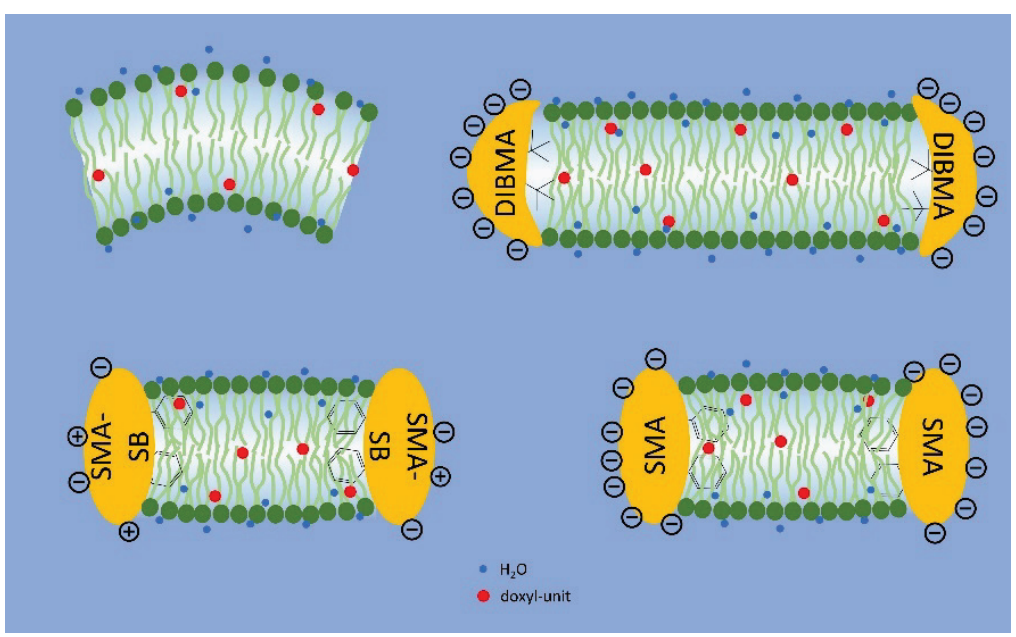


Figure 3 Schematic comparison of the local structures in DMPC liposomes (upper left-hand side) and the three nanodisc systems SMALPs, DIBMALPs, and SMA-SBLPs. The location of the doxyl units is highlighted as red dots as well as the respective side groups of the polymer chain that reach inside the lipid bilayer system. Differences in water penetration of all bilayer systems are indicated by exemplary water molecules (blue dots).

\section{Conclusions}

In this work, we analyzed CW EPR spectra of 5DSPC and 16DSPC incorporated in DMPC nanodiscs and compared them to both spin-labeled lipids in liposomes. We found that:

- all polymers induced broadening of the DMPC main transition while maintaining a similar $T_{\mathrm{m}}$,

- with all polymers, the water accessibility of the near-headgroup region and the center of the membrane changed,

- the segmental order parameter was increased in all studied nanodisc systems compared with LUVs, and

- $S_{z z}$ of DMPC in DIBMALPs appeared to be slightly less affected than with both other polymers probably due to DIBMA not bearing phenyl groups.

All of these observations are schematically summarized in Figure 3. They indicate that the polymers exhibit different influences on the polar and apolar lipid regions, which should be considered when preparing polymer-solubilized lipids for protein studies or other in vitro investigations involving lipidic model membranes. CW EPR has long been known to be a valuable method to obtain insights into the ordering, rotational mobility, and water accessibility of lipids in large vesicles and we here show that it can even help understand slight differences in the local nanodisc structure induced by different LPforming polymers. While DSC measurements indicate a rather small macroscopic impact of the polymers on the lipids, several effects exerted by the different polymers on the level of the lipid molecules were observed with CW EPR spectroscopy, which allows characterization of heterogeneity in lipid mobility and local structuring.

\section{Associated Content}

The Supporting Information is available online. (Nanodisc characterization with DLS/TEM, CW EPR simulation routine, comparison of simulated and measured spectra, further simulation results)

\section{Author Information}

Corresponding author:

E-Mail: dariush.hinderberger@chemie.uni-halle.de; phone: +49-345-5525230

Author contributions:

M.H., J.E., F.S., C.V., S.K., and D.H. conceived and planned research; M.H. and M.D. prepared samples; M.H., J.E., and F.S. performed experiments; M.H., J.E., F.S., and D.H. analyzed data; J.E. and F.S. carried out spectral simulations; M.H., J.E., F.S., and D.H. wrote the publication.

\section{Declaration of competing interest:}

The authors declare no competing financial interest.

\section{Acknowledgments}


M.H. thanks the German Chemical Industry Association (VCl) for financial support in form of the Kekule scholarship. J.E. thanks the Alexander von Humboldt foundation for financial support through a Feodor Lynen Research Fellowship. This work was supported by the Deutsche Forschungsgemeinschaft (DFG) in Research Training Group (RTG) 2670, "Beyond Amphiphilicity".

We thank Annekatrin Rother and Gerd Hause for their support in obtaining TEM micrographs. We thank PD Dr. Annette Meister for access to a plasma cleaner and general helpful discussions.

\section{References}

[1] J.M. Dörr, S. Scheidelaar, M.C. Koorengevel, J.J. Dominguez, M. Schäfer, C.A. van Walree, J.A. Killian, The styrene-maleic acid copolymer: a versatile tool in membrane research, Eur. Biophys. J., 45 (2016) 3-21.

[2] S.C. Lee, N.L. Pollock, Membrane proteins: is the future disc shaped?, Biochem. Soc. Trans., 44 (2016) 1011-1018.

[3] N. Österlund, J. Luo, S.K.T.S. Wärmländer, A. Gräslund, Membrane-mimetic systems for biophysical studies of the amyloid-beta peptide, Biochim. Biophys. Acta. Proteins Proteom., 1867 (2019) 492-501.

[4] M. Overduin, M. Esmaili, Structures and Interactions of Transmembrane Targets in Native Nanodiscs, SLAS Discov., 24 (2019) 943-952.

[5] T.J. Knowles, R. Finka, C. Smith, Y.P. Lin, T. Dafforn, M. Overduin, Membrane Proteins Solubilized Intact in Lipid Containing Nanoparticles Bounded by Styrene Maleic Acid Copolymer, J. Am. Chem. Soc., 131 (2009) 7484-7485.

[6] A.O. Oluwole, B. Danielczak, A. Meister, J.O. Babalola, C. Vargas, S. Keller, Solubilization of Membrane Proteins into Functional Lipid-Bilayer Nanodiscs Using a Diisobutylene/Maleic Acid Copolymer, Angew. Chem. Int. Ed., 56 (2017) 1919-1924.

[7] S. Scheidelaar, M.C. Koorengevel, J.D. Pardo, J.D. Meeldijk, E. Breukink, J.A. Killian, Molecular model for the solubilization of membranes into nanodisks by styrene maleic acid copolymers, Biophys. J., 108 (2015) 279-290.

[8] S. Scheidelaar, M.C. Koorengevel, C.A. van Walree, J.J. Dominguez, J.M. Dörr, J.A. Killian, Effect of Polymer Composition and $\mathrm{pH}$ on Membrane Solubilization by Styrene-Maleic Acid Copolymers, Biophys. J., 111 (2016) 1974-1986.

[9] B. Danielczak, A. Meister, S. Keller, Influence of $\mathrm{Mg}(2+)$ and $\mathrm{Ca}(2+)$ on nanodisc formation by diisobutylene/maleic acid (DIBMA) copolymer, Chem. Phys. Lipids, 221 (2019) 30-38.

[10] A.O. Oluwole, J. Klingler, B. Danielczak, J.O. Babalola, C. Vargas, G. Pabst, S. Keller, Formation of Lipid-Bilayer Nanodiscs by Diisobutylene/Maleic Acid (DIBMA) Copolymer, Langmuir, 33 (2017) 1437814388.

[11] A. Grethen, A.O. Oluwole, B. Danielczak, C. Vargas, S. Keller, Thermodynamics of nanodisc formation mediated by styrene/maleic acid (2:1) copolymer, Sci. Rep., 7 (2017) 11517.

[12] R. Cuevas Arenas, J. Klingler, C. Vargas, S. Keller, Influence of lipid bilayer properties on nanodisc formation mediated by styrene/maleic acid copolymers, Nanoscale, 8 (2016) 15016-15026.

[13] T. Ravula, S.K. Ramadugu, G. Di Mauro, A. Ramamoorthy, Bioinspired, Size-Tunable Self-Assembly of Polymer-Lipid Bilayer Nanodiscs, Angew. Chem. Int. Ed., 56 (2017) 11466-11470.

[14] A.A.A. Smith, H.E. Autzen, B. Faust, J.L. Mann, B.W. Muir, S. Howard, A. Postma, A.J. Spakowitz, Y. Cheng, E.A. Appel, Lipid Nanodiscs via Ordered Copolymers, Chem., 6 (2020) 2782-2795.

[15] J. Eisermann, M. Hoffmann, F.A. Schöffmann, M. Das, C. Vargas, S. Keller, D. Hinderberger, Molecular-level interactions of nanodisc-forming copolymers dissected by EPR spectroscopy, Macromol. Chem. Phys., accepted manuscript (2021).

[16] W. Mosslehy, N. Voskoboynikova, A. Colbasevici, A. Ricke, D. Klose, J.P. Klare, A.Y. Mulkidjanian, H.J. Steinhoff, Conformational Dynamics of Sensory Rhodopsin II in Nanolipoprotein and StyreneMaleic Acid Lipid Particles, Photochem. Photobiol., 95 (2019) 1195-1204.

[17] J.J. Dominguez Pardo, J.M. Dörr, M.F. Renne, T. Ould-Braham, M.C. Koorengevel, M.J. van Steenbergen, J.A. Killian, Thermotropic properties of phosphatidylcholine nanodiscs bounded by styrene-maleic acid copolymers, Chem. Phys. Lipids, 208 (2017) 58-64. 
[18] P. Stepien, A. Polit, A. Wisniewska-Becker, Comparative EPR studies on lipid bilayer properties in nanodiscs and liposomes, Biochim. Biophys. Acta, 1848 (2015) 60-66.

[19] M. Isabel Collado, F.M. Goni, A. Alonso, D. Marsh, Domain Formation in Sphingomyelin/Cholesterol Mixed Membranes Studied by Spin-Label Electron Spin Resonance Spectroscopy, Biochemistry, 44 (2005) 4911-4918.

[20] S. Stoll, A. Schweiger, EasySpin, a comprehensive software package for spectral simulation and analysis in EPR, J. Magn. Reson., 178 (2006) 42-55.

[21] A. Colbasevici, N. Voskoboynikova, P.S. Orekhov, M.E. Bozdaganyan, M.G. Karlova, O.S. Sokolova, J.P. Klare, A.Y. Mulkidjanian, K.V. Shaitan, H.J. Steinhoff, Lipid dynamics in nanoparticles formed by maleic acid-containing copolymers: EPR spectroscopy and molecular dynamics simulations, Biochim. Biophys. Acta Biomembr., 1862 (2020) 183207.

[22] D. Marsh, Distinct Populations in Spin-Label EPR Spectra from Nitroxides, J. Phys. Chem. B, 122 (2018) 6129-6133.

[23] S. Ali, S. Minchey, A. Janoff, E. Mayhew, A Differential Scanning Calorimetry Study of Phosphocholines Mixed with Paclitaxel and Its Bromoacylated Taxanes, Biophys. J., 78 (2000) 246-256. [24] D.A. Erilov, R. Bartucci, R. Guzzi, A.A. Shubin, A.G. Maryasov, D. Marsh, S.A. Dzuba, L. Sportelli, Water Concentration Profiles in Membranes Measured by ESEEM of Spin-Labeled Lipids, J. Phys. Chem. B, 109 (2005) 12003-12013.

[25] D. Kurad, G. Jeschke, D. Marsh, Lipid Membrane Polarity Profiles by High-Field EPR, Biophys. J., 85 (2003) 1025-1033.

[26] M.R. Vartorelli, A.S. Garay, D.E. Rodrigues, Spin-labeled Stearic Acid Behavior and Perturbations on the Structure of a Gel-Phase-Lipid Bilayer in Water: 5-, 12- and 16-SASL, J. Phys. Chem. B, 112 (2008) 16830-16842.

[27] H.J. Steinhoff, Multi-Frequency EPR Spectroscopy Studies of the Structure and Conformational Changes of Site-Directed Spin Labelled Membrane Proteins, in: G. Pifat-Mrzljak (Ed.) Supramolecular Structure and Function, vol. 8, Springer, New York, 2004, pp. 157-177. 